\title{
Montecarlo simulation of the High Energy Particle Detector on board the satellite CSES
}

\author{
Luca Carfora for the CSES/HEPD collaboration ${ }^{1, a}$ \\ ${ }^{1}$ University of Rome "Tor Vergata", Department of Physics, Via della Ricerca Scientifica 1 - 00133 Rome \\ (Italy)
}

\begin{abstract}
The High-Energy Particle Detector (HEPD) is an instrument devoted to the measurement of cosmic particles from few $\mathrm{MeV}$ up to hundreds of $\mathrm{MeV}$. The HEPD will contribute to the China Seismo-Electromagnetic Satellite mission by measuring the precipitation of trapped particles and by studying the solar-terrestrial environment especially during impulsive events like coronal mass ejections and solar energetic particle emissions.

A Monte Carlo software was realized to study the performance of HEPD, such as its particle discrimination capability, the energy threshold for trigger and the maximum energy detectable in full containment.

This contribution reports the main features of the HEPD Monte Carlo simulation and some results of the detector performance based on it. A comparison with beam tests was carried out, showing a good agreement with the simulation.
\end{abstract}

\section{HEPD: High Energy Particle Detector}

The HEPD was developed by the Italian "Limadou" collaboration in the framework of the ChineseItalian CSES mission.

The detector will range the low-energy component of cosmic rays, and in particular electrons in the range $3-100 \mathrm{MeV}$, protons in the range $30-300 \mathrm{MeV}$ and light nuclei. It is composed by several subdetectors, arranged as shown in Fig. 1, left: a tracker, with 2 planes of double-sided silicon microstrip detectors; a trigger plane, formed by 6 plastic scintillator paddles; a range-calorimeter, a tower of 16 plastic scintillator planes and a $3 \times 3$ matrix of inorganic scintillator (LYSO); with the tracker/trigger plane it is used for the particle identification and the energy reconstruction. A veto system based on a set of plastic scintillators rejects events that are not fully contained in the calorimeter.

HEPD is installed on board the CSES satellite, a scientific mission dedicated to monitor electromagnetic field and waves, plasma and particles perturbations of the atmosphere, ionosphere and magnetosphere induced by natural sources and anthropocentric emitters [1] and to study their correlations with the occurrence of seismic events [2][3]. Data collected by the mission will also allow studying solar-terrestrial interactions and cosmic ray solar modulation, with particular regard to particle spectra of the 24th and 25th solar cycle.

a e-mail: luca.carfora@roma2.infn.it 

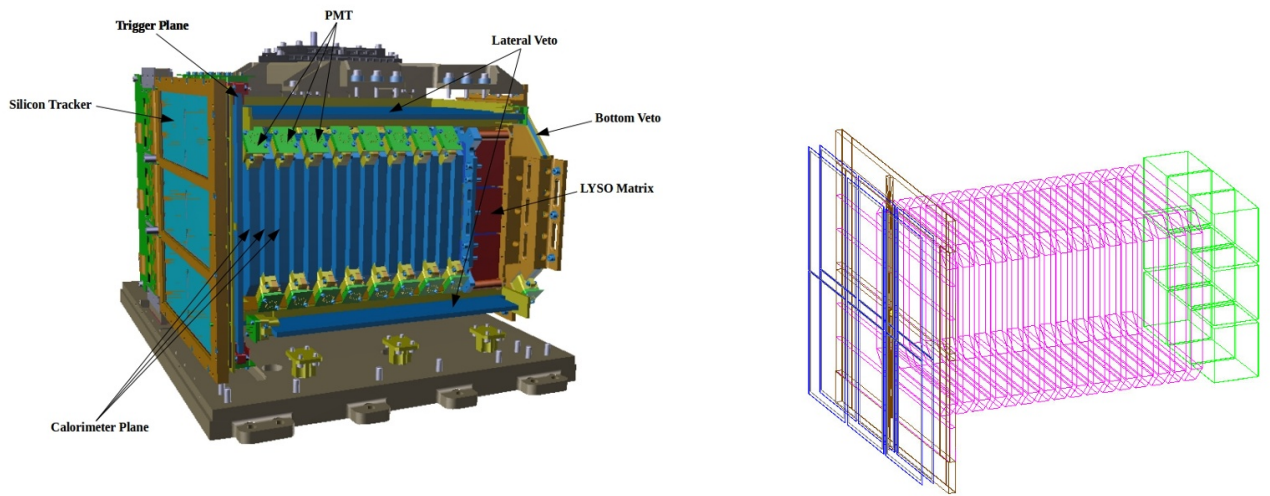

Figure 1. Left: An overview of HEPD. The trigger plane, below the silicon planes, is not visible. Right: example of the graphic transparent visualization of HEPD MC software; veto planes, HEPD box, satellite wall and passive materials are not shown.

\section{HEPD MC simulation}

The performance of HEPD using different particles and energies was studied with a Monte Carlo software, based on Geant 4 package. Geant 4 is a toolkit for the simulation of the passage of particles through matter. Its areas of application are in high energy, nuclear and accelerator physics and studies in medical and space science [4] [5] [6].

HEPD geometry is reported in Fig. 1, right. Different colours are used for different sub-detectors (blue for the silicon tracker, magenta for all the plastic scintillators and green for the LYSO crystals). The veto system and all mechanical structures have been hidden in the figure.

In the HEPD MC software there is the possibility of reproducing different environments, such as the beam test configurations, the muon configuration (atmospheric muons simulated with angular and energy distribution according to [7]) and the flight configuration. The production of optical photons inside the scintillators and the light collected by each PMT has also been added to the simulation.

\subsection{Trigger energy threshold and maximum energy range}

A simulation was carried out to study the particle energy thresholds with different trigger configurations and with maximum energy detectable, requesting in this case that a particle is fully contained in HEPD. The simulated data set consisted in 1 million of electrons and 1 million of protons with a uniform energy spectrum respectively between 1 and $100 \mathrm{MeV}$ and 1 and $300 \mathrm{MeV}$ and an angular spectrum uniform in $\cos ^{2} \theta$.

Energy ranges have been studied with several selections on these events (shown in Figure 2):

- the minimum limit in Fig. 2 (a) and (b) is realized with different trigger configurations (some of which used in flight);

- the maximum range is evaluated as the energy of events fully contained in the calorimeter; the overall selection is one trigger paddle hit and no lateral veto hit (Fig. 2 (c) and (d)). 

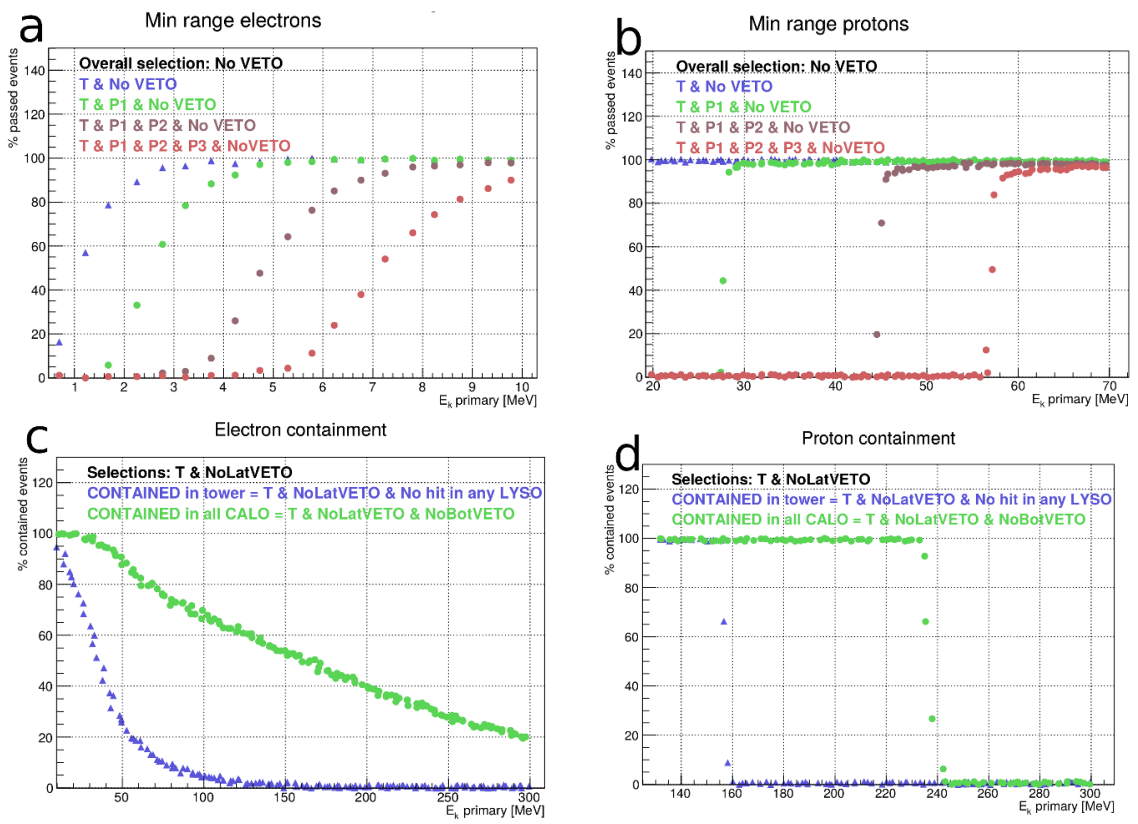

Figure 2. Left: Fraction of electrons (a) and of protons (b) that pass some trigger conditions as a function of primary energy; Right: fraction of electrons (c) and of protons (d) contained in the tower and in the whole calorimeter as a function of primary energy.

With a trigger configuration of one trigger paddle and the first two planes hit, electron minimum detectable energy is $6 \mathrm{MeV}$ with $80 \%$ probability with respect to the overall events; for protons, it is $45 \mathrm{MeV}$. The maximum energy curve for electrons is not sharp because of Bremsstrahlung photons produced by electrons in scintillator material. Maximum energy for a proton contained in the calorimeter is $240 \mathrm{MeV}$.

\subsection{Particle discrimination}

Using the same data set described above, the particle identification was studied with the $\Delta \mathrm{E}$ vs $\mathrm{E}$ method.

Fig. 3 shows the $\Delta \mathrm{E}$ vs $\mathrm{E}$ method for particle discrimination, where $\Delta \mathrm{E}$ is the energy deposit in one thin thickness and $\mathrm{E}$ is the total energy deposit measured by the detector. Two particle populations (protons on the top and electrons on the bottom) are clearly visible.

\subsection{MC digitization}

Digitization of the MC data consists in the conversion of the light collected by each PMT into a signal in ADC counts. The digitization factors were obtained comparing the peak and sigma values of ADC signals of PMTs recorded during proton test beams at different energies with distributions of light collected from PMTs in the corresponding HEPD simulations.

Both data and MC distributions were realized with the same selection: 


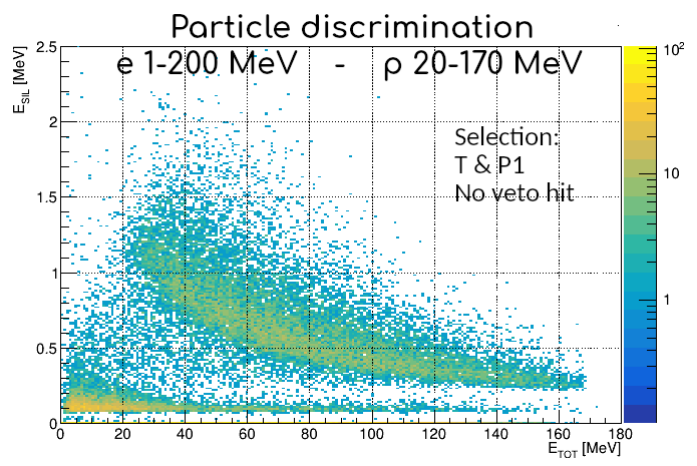

Figure 3. $\Delta \mathrm{E}$ vs $\mathrm{E}$ method for particle identification with a sample of simulated electrons and protons; in the plot, $E_{S I L}$ is the energy deposit in the $1^{\text {st }}$ tracker plane, $E_{T O T}$ is the energy deposit in the detector.
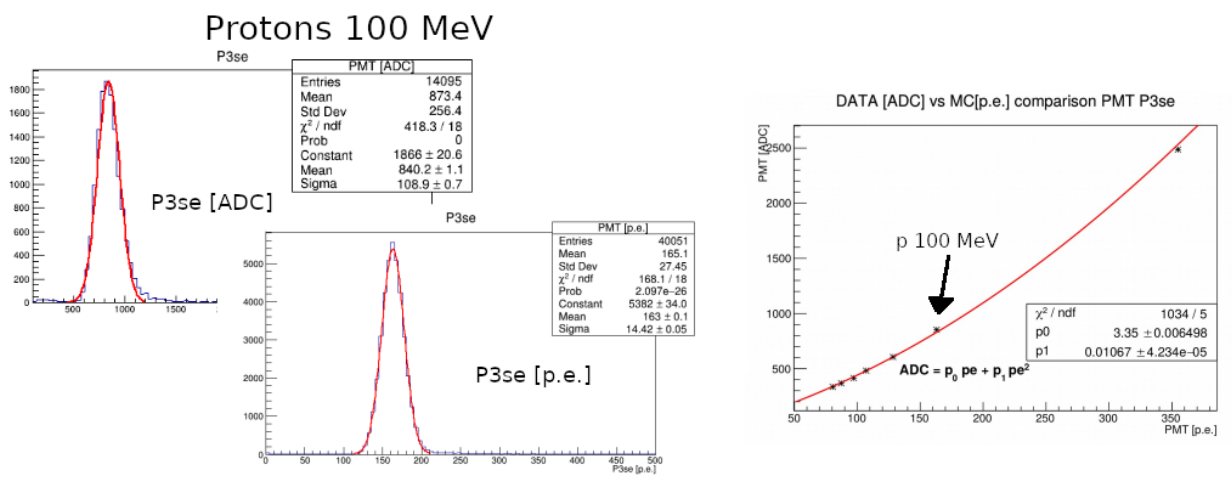

Figure 4. Left: distributions of ADC signal and p.e. (photo electrons) from PMT P3se in the $100 \mathrm{MeV}$ proton run; both distributions were fitted with a gaussian; Right: peak of ADC signal as a function of light collected from PMT P3se in all proton runs; the curve was fitted with a parabola.

- most significant trigger paddle \& P1 (both PMTs over 10 $\sigma$ );

- no hit in any veto (both PMTs under $5 \sigma$ );

- trigger multiplicity equal to 1 (both PMTs under $5 \sigma$ ).

Values of the peak distributions were correlated in ADC-p.e. (photo electrons) plots (one for each PMT) and points were fitted with a function of the type: $A D C=p_{0} \cdot P E+p_{1} \cdot P E^{2}$. ADC is the signal pedestal-subtracted.

Digitizaton factors $p_{0}$ and $p_{1}$ were applied to MC light distributions to verify the correctness of the procedure; the comparison between real and reconstructed ADC distributions for some PMTs for protons and for a set of independent data (electrons beam test and muons) is shown in fig. 5. 

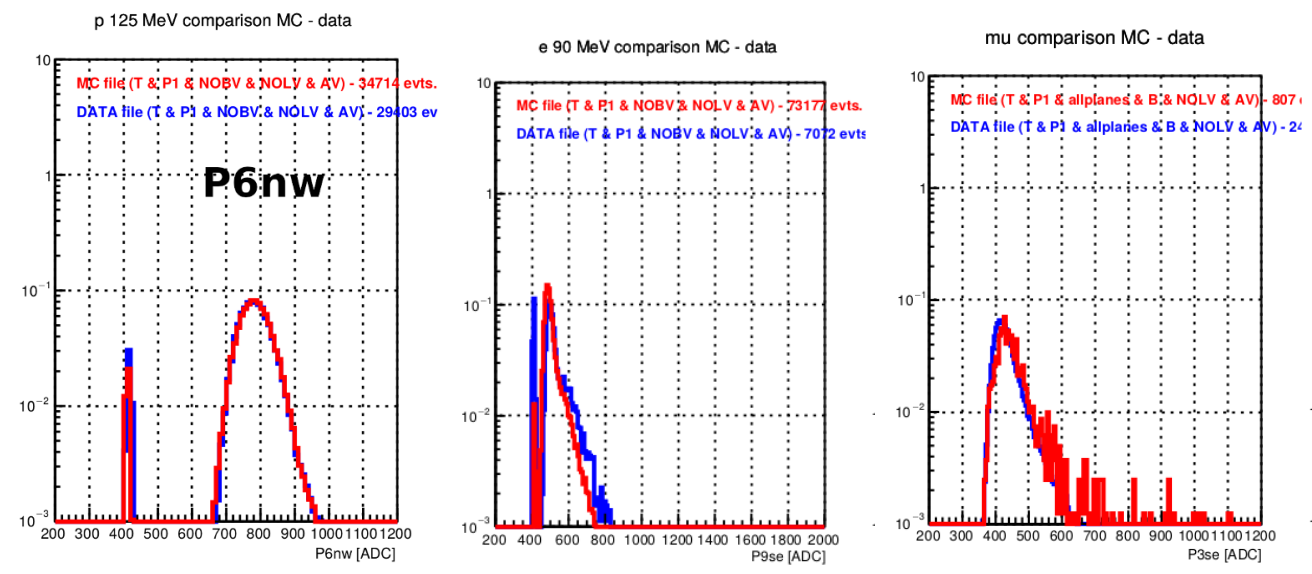

Figure 5. Comparison between distributions of real (blue) and reconstructed ADC signal from MC (red) for 3 PMTs for $125 \mathrm{MeV}$ protons (left), $90 \mathrm{MeV}$ electrons (center) and muons (right). Selections used are the same for the two distributions and are: most significant paddle \& P1, no hit in any veto and trigger multiplicity equal to 1.

\section{Conclusions}

In this contribution some results of HEPD Monte Carlo simulation (energy range, particle discrimination) were presented; they show a good agreement with test beam and flight data analysis.

Furthermore, the digitization procedure for PMT scintillator signals was described. It will be used to support the equalization of PMTs and the calibration of the calorimeter.

\section{References}

[1] V. Vitale et al. (CSES/HEPD collaboration), EPJ Web of Conferences 136, 4 (2017)

[2] X. Shen et al., Earthquake Science 28, 303 (2015)

[3] A. De Santis et al., Physics and Chemisrty of the Earth 85-86, 1 (2015)

[4] A. S. et al., Nucl. Instr. and Meth. in Phys. Res. A 506, 250 (2003)

[5] J. Allison et al., IEEE Transactions on Nucl. Sc. 53, 270 (2006)

[6] J. Allison et al., Nucl. Instr. and Meth. in Phys. Res. A 835, 186 (2016)

[7] S. Cecchini, M. Spurio, Geoscientific Instrumentation Methods and Data System 1, 185 (2012) 